\title{
Changes in Treatment Patterns and Incremental Health Care Utilization Due to P2Y12-Associated Complications in Patients with Acute Coronary Syndrome
}

\author{
Ami Vyas, PhD, MS; Lori D. Bash, PhD, MPH; Mehul D. Patel, PhD; and Ross J. Simpson, Jr., MD, PhD
}

\section{ABSTRACT}

BACKGROUND: P2Y12 antiplatelet therapy (APT) is highly efficacious in reducing the incidence of ischemic events in patients with acute coronary syndrome (ACS); however, it is associated with several adverse complications. Data on P2Y12-associated complications and adherence to APT are sparse.

OBJECTIVE: To describe the characteristics, frequency of P2Y12-associated complications, adherence and persistence to P2Y12 APT, and health care utilization among ACS patients on P2Y12 APT.

METHODS: This retrospective observational study of the MarketScan Commercial Claims and Encounters Database identified patients aged $\geq 18$ years who were discharged from an ACS hospitalization in 2012-2014 and initiated P2Y12 APT (ticagrelor, prasugrel, or clopidogrel). The proportion of patients within each treatment group who experienced P2Y12-associated complications within 1 year and who were adherent to APT were determined. Frequencies of all-cause health care utilization (i.e., hospitalization, length of stay, emergency room [ER] visits, outpatient visits, cardiac events, and transfusions) were evaluated for each treatment group. Poisson regressions were conducted to evaluate the association between nonadherence with P2Y12 APT and health care utilization, after adjusting for demographics (age and gender), health insurance type, and comorbidities.

RESULTS: Among 11,629 ACS patients, most were male; $44.6 \%$ had hypertension; $20.6 \%$ had diabetes; and $53.4 \%$ had hyperlipidemia. Clopidogrel use was common (62.6\%), with ticagrelor use less common (9.0\%). Among all groups, approximately one third experienced P2Y12-associated complications. One-year adherence to APT was suboptimal ( $68 \%$ overall), with $73.3 \%$ adherence among prasugrel users, followed by $71.4 \%$ adherence among ticagrelor users and $65.6 \%$ adherence among clopidogrel users. Switching was most common with ticagrelor users. Inpatient hospitalizations, cardiac events, and transfusions were more common in clopidogrel users compared with prasugrel and ticagrelor users. Nonadherent patients experienced significantly more hospitalizations, ER visits, and transfusions (1.34, 1.09, and $1.85[P<0.05]$, respectively) compared with adherent patients. These trends of association remained consistent across all treatment groups. Also, patients not adherent to ticagrelor experienced 1.9 times as many cardiac events as adherent patients. However, this association was not significant for clopidogrel and prasugrel users. Patients not adherent to P2Y12 APT experienced significantly lower outpatient visits compared with adherent patients.

CONCLUSIONS: Complications associated with P2Y12 in ACS patients treated with P2Y12 APT were common, with dyspnea, heart block, and major or life-threatening bleeding as the most common. Adherence was significantly associated with lower health care utilization. Increased adherence to secondary prevention therapy among these very high-risk patients is crucial. Disease management strategies to improve adherence and reduce treatment-associated adverse events through individualized patient care, alternative secondary treatment options, and physician awareness should be designed, implemented, and sustained.

\section{J Manag Care Spec Pharm. 2017;23(9):947-56}

Copyright $\odot 2017$, Academy of Managed Care Pharmacy. All rights reserved.

\section{What is already known about this subject}

Although P2Y12 antiplatelet therapy (APT) is highly efficacious in reducing the incidence of ischemic events, it is highly associated with complications that may result in drug discontinuation, which affect health care utilization.

A real-world study has confirmed a high rate of drug withdrawal in patients with acute coronary syndrome (ACS) who were treated with ticagrelor; however, the scope of the study was limited in both treatment and population.

\section{What this study adds}

This study assessed the proportion of ACS patients who experienced P2Y12-associated complications and adherence and persistence to P2Y12 APT, along with its association with all-cause health care utilization, using a large U.S. commercial claims database.

More patients treated with clopidogrel had inpatient hospitalizations, cardiac events, and transfusions compared with ticagrelor users and prasugrel users.

Adherence to P2Y12 APT was suboptimal, and better adherence was highly associated with lower hospitalizations, emergency room visits, and transfusions.

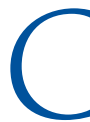

oronary heart disease remains a significant burden in developed and developing countries. Globally, cardiovascular disease is the major cause of death, accounting for over 17.5 million deaths (31\% of the all global deaths) in 2012. ${ }^{1}$ Acute coronary syndrome (ACS) is the initial presentation of a patient manifesting coronary artery disease. It is estimated that each year in the United States more than 780,000 people experience ACS. In 2010, ACS resulted in more than 1.14 million hospitalizations. ${ }^{2}$

To prevent any recurrent ischemic event for ACS patients, dual antiplatelet therapy with a combination of aspirin and a 
P2Y12 inhibitor (clopidogrel, prasugrel, or ticagrelor) is recommended for a period of 12 months., ${ }^{3,4}$ Although clopidogrel is generally well tolerated in ACS patients, the irreversible nature of its antiplatelet action has raised concerns of increased bleeding risk, including a nonsignificant increase in life-threatening and fatal bleeding, severe and moderate bleeding, and dyspnea..$^{5-7}$ A third-generation drug, prasugrel, is found to be effective as well, but there are also concerns regarding the higher bleeding risk associated with this drug. ${ }^{8-11}$ The frequencies of Thrombolysis in Myocardial Infarction (TIMI) major or minor bleeding were higher, and fatal hemorrhage was more frequent with prasugrel than with clopidogrel. ${ }^{8}$

In the PLATelet inhibition and patient Outcomes (PLATO) trial, a relatively new P2Y12 receptor inhibitor, ticagrelor 90 $\mathrm{mg}$, has shown better health outcomes in terms of morbidity and mortality, compared with clopidogrel in patients with ACS, although it is associated with increased incidence of intracranial bleeding, fatal intracranial bleeding, major or minor bleeding, dyspnea and ventricular pauses, syncope, heart block, and increase in level of serum uric acid and serum creatinine. ${ }^{12}$ In addition, a recently published trial that assessed the benefit of ticagrelor $60 \mathrm{mg}$ and $90 \mathrm{mg}$, both twice daily, in patients 1-3 years post-ACS reported nearly similar efficacy in reducing the risk of cardiovascular death, myocardial infarction, or stroke, although this benefit was associated with higher TIMI major or minor bleeding and serious dyspnea compared with placebo. ${ }^{13}$ However, the U.S. Food and Drug Administration and the European Medicines Agency approved and granted marketing authorization for the lower $60 \mathrm{mg}$ dose for long-term use of ticagrelor after it demonstrated equal efficacy and an improved safety profile compared with the $90 \mathrm{mg}$ dose. ${ }^{13-15}$

Although P2Y12 antiplatelet therapy (APT) is highly efficacious in reducing the incidence of ischemic events when used, it is associated with several adverse reactions, referred to as P2Y12-associated complications, including dyspnea, major or life-threatening bleeding, major gastrointestinal bleeding, bradycardia, heart block, and syncope. These P2Y12-associated complications may lead to higher discontinuation of APT by patients, which may ultimately affect treatment effectiveness and health care utilization. A real-world observational study that evaluated the effect of ticagrelor-related dyspnea on compliance with therapy in ACS patients reported that the rate of drug withdrawal was high, and drug-related dyspnea was the main reason for noncompliance with therapy followed by drug-related minor bleeding. ${ }^{16}$ However, this study was limited to only 1 P2Y12 antiplatelet drug (ticagrelor), focused on only 1 associated complication (dyspnea), and was geographically limited to a small French population.

Additional real-world information about P2Y12-associated complications in ACS patients and their effects on adherence to P2Y12 APT and consequent incremental health care utilization is much needed. A better understanding of patient characteristics, adherence and persistence to APT, and its association with incremental health care utilization in ACS patients in the real-world setting could inform treatment guidelines and aid providers in clinical decision making

The purpose of this study was to (a) assess the frequency of P2Y12-associated complications in ACS patients, along with their adherence and persistence to P2Y12 APT, in a large U.S. claims database, and (b) evaluate the association of nonadherence to P2Y12 APT with health care utilization in these patients.

\section{Methods}

\section{Study Design and Data Source}

This retrospective observational cohort study used the MarketScan Commercial Claims and Encounters Database (MCED), a HIPPA-compliant, employer-sponsored health care claims database. This database has covered approximately 200 million unique patients since 1995 and includes insured employees, early retirees, COBRA continuers, and their dependents from approximately 100 large, employer-sponsored health plans, as well as those who are Medicare eligible but choose to have supplemental employer-based insurance. The database contains enrollees residing in all 50 states and the District of Columbia. Although representation is greater from the South, the data are broadly representative of the United States as a whole. ${ }^{17}$ The MCED combines 2 separate databases: Commercial Claims and Encounters Database, and the Medicare Supplemental and Coordination of Benefit Database. The data components used in this study included health plan enrollment records, participant demographics, inpatient and outpatient medical services, and outpatient prescription drug dispensing records.

\section{Study Cohort}

The study cohort consisted of patients aged $\geq 18$ years who were discharged from an ACS hospitalization between January 1, 2012, and December 31, 2014, and who initiated index P2Y12 APT (ticagrelor, prasugrel, or clopidogrel) within 30 days of their discharge. ACS was identified from the inpatient claims and defined as a primary diagnosis code from the International Classification of Diseases, Ninth Revision, Clinical Modification (ICD-9-CM) code 410.xx (excluding 410.x2). The date of discharge for the ACS hospitalization was considered the index date. Eligible patients were required to be alive at discharge and have at least 12 months of baseline continuous enrollment before the index ACS hospitalization and at least 12 months of follow-up after the index event.

Patients who initiated more than 1 P2Y12 APT at the same time and who received P2Y12 APT for greater than 30 days or other oral anticoagulation therapy (except aspirin) during the baseline period were excluded. Use of P2Y12 APT was identified from outpatient pharmacy claims using all National Drug Code numbers associated with the respective generic names. 
The final study sample was analyzed across 3 treatment groups based on the type of P2Y12 antiplatelet medication initiated after ACS hospitalization.

\section{Measures}

Baseline Characteristics. Baseline characteristics included basic demographics (age and gender), health insurance plan type, comorbidities, and concomitant prescription medications. Comorbidities were identified from medical claims from the 12 months before the index event. Concomitant prescription medications were assessed during the baseline period.

Complications Associated with P2Y12. Patients meeting the inclusion criteria were followed up to 12 months for episodes of P2Y12-associated complications. These complications included dyspnea, bradycardia, syncope, heart block, elevated serum creatinine and uric acid levels, intracranial bleeding or hemorrhage, major or life-threatening bleeding, and major gastrointestinal bleeding. ${ }^{18}$ These episodes were identified using the first claim originating from an emergency room (ER), inpatient claim, outpatient claim, or physician services claim with the respective ICD-9-CM codes as listed in Appendix A (available in online article).

P2Y12 Treatment Changes. During the follow-up period, switch, or discontinuation in therapy, adherence and persistence to the index P2Y12 APT were assessed. A switch in therapy was defined as patients who discontinued use of a current P2Y12 APT for 30 days or more and replaced it with another antiplatelet drug. Adherence is the degree to which a patient remains compliant to the prescribed medication and is operationally defined as the proportion of days covered (PDC) and calculated as the ratio of number of days P2Y12 APT was supplied and number of days in the follow-up period. ${ }^{19}$ Patients were considered to be adherent to APT if their PDCs were $\geq 80 \%$.

Persistence is the patient's duration from medication initiation to discontinuation and is operationalized dichotomously, defined as continuation or discontinuation of P2Y12 APT where the gap in therapy of at least 30 days (between the refill date and the calculated run-out date of previous medication) was considered discontinuation and hence nonpersistence to the therapy. Nonpersistence during the follow-up period was defined as the first incident of failure to refill index medication within 30 days from the run-out date of the previous prescription. ${ }^{20}$

Health Care Utilization. The measures of all-cause health care utilization included frequencies of all-cause inpatient hospitalization, ER visits, outpatient visits, cardiac events, and transfusions. In addition, length of hospital stay in days was also assessed. Cardiac events were identified from inpatient claims and were defined as having had acute myocardial infarction, which was identified using the ICD-9-CM code 410.xl, or having had revascularization, identified using the ICD-9-CM procedure codes $36.00,36.06,36.07,36.09$, and 36.10-36.19 during the follow-up period. Transfusions were identified from the inpatient claims using ICD-9-CM code 99.xx.

\section{Statistical Analyses}

Descriptive statistics of all patient demographic and clinical characteristics were conducted for the overall population, as well as for index treatment groups. Chi-square tests for categorical variables and t-tests or Kruskal-Wallis tests for continuous variables were used to assess significant differences between the treatment groups. The proportion of total patients and patients within treatment groups who experienced P2Y12associated complications were determined. The proportion of patients who were adherent and persistent to APT and who switched to another APT were also estimated. Frequencies of hospitalizations, outpatient visits, ER visits, cardiac events, and transfusions, along with length of hospital days within each treatment group, were estimated and reported as mean (standard deviations [SDs]) or median (interquartile range [IQR]). Multivariate Poisson regressions were performed to evaluate if nonadherence with P2Y12 APT was associated with health care utilization within each treatment group. $P$ values $<0.05$ were considered statistically significant. All statistical analyses were performed using SAS software version 9.4 (SAS Institute, Cary, NC).

\section{Results}

\section{Baseline Characteristics}

Of the 143,238 patients hospitalized for ACS between January 1 , 2012, and December 21, 2014, 11,629 met the inclusion criteria (Appendix B, available in online article). Patients were more often men (76.2\%) and, on average, aged 57 years (Table 1). The majority $(71.2 \%)$ of the study cohort was aged between 45 years and 64 years and had preferred provider organization insurance coverage (53.4\%). Comorbid hyperlipidemia, hypertension, diabetes, and previous myocardial infarction were common $(53.4 \%, 44.6 \%, 20.6 \%$, and $42.2 \%$, respectively) among these patients. The vast majority were taking prescription beta-adrenergic antagonists (73.3\%) and statins (77.7\%), while $49.5 \%$ were on angiotensin-converting enzyme inhibitors.

Among ACS patients treated with P2Y12, 9.0\% initiated ticagrelor; $28.4 \%$ initiated prasugrel; and $62.6 \%$ initiated clopidogrel within 30 days of discharge. Treatment groups significantly differed across most baseline characteristics (Table 1), with the exception of exclusive provider organizations or health maintenance organizations and point-of-service insurance plans, previous myocardial infarction and coronary revascularization, peripheral arterial disease, and use of angiotensin II receptor antagonists and proton pump inhibitors.

\section{Complications Associated with P2Y12}

Of the 11,629 patients who met the inclusion criteria, 33\% experienced P2Y12-associated complications during the 
TABLE 1 Baseline Characteristics of ACS Patients Who Initiated P2Y12 APT Within 30 Days of ACS Event

\begin{tabular}{|c|c|c|c|c|c|c|c|c|c|c|c|}
\hline \multirow[b]{2}{*}{ Variables } & \multirow[b]{2}{*}{\begin{tabular}{|c|} 
All \\
Patients \\
$\mathrm{N}=11,629$ \\
\end{tabular}} & \multicolumn{3}{|c|}{ Index P2Y12 Treatment } & \multirow[b]{2}{*}{$P$ Value } & \multirow[b]{2}{*}{ Variables } & \multirow[b]{2}{*}{$\begin{array}{c}\text { All } \\
\text { Patients } \\
\mathrm{N}=11,629\end{array}$} & \multicolumn{3}{|c|}{ Index P2Y12 Treatment } & \multirow[b]{2}{*}{$P$ Value } \\
\hline & & $\begin{array}{c}\text { Ticagrelor } \\
\text { Users } \\
\mathbf{n}=1,049\end{array}$ & $\begin{array}{c}\text { Prasugrel } \\
\text { Users } \\
n=3,297\end{array}$ & $\begin{array}{c}\text { Clopidogrel } \\
\text { Users } \\
\mathbf{n}=7,283\end{array}$ & & & & $\begin{array}{c}\text { Ticagrelor } \\
\text { Users } \\
n=1,049\end{array}$ & $\begin{array}{c}\text { Prasugrel } \\
\text { Users } \\
\mathbf{n}=3,297\end{array}$ & $\begin{array}{c}\text { Clopidogrel } \\
\text { Users } \\
\mathbf{n}=7,283\end{array}$ & \\
\hline Age, mean (SD) & $57.4(11.0)$ & $56.1(9.7)$ & $54.6(8.5)$ & $58.9(11.9)$ & $<0.0001$ & \multicolumn{6}{|c|}{ Comorbidities, \% } \\
\hline \multicolumn{6}{|c|}{ Age group, years, $\%$} & Coronary & 6.9 & 7.6 & 7.0 & 6.8 & 0.6260 \\
\hline$<35$ & 0.9 & 1.0 & 0.8 & 0.9 & 0.9091 & revasc ${ }^{a}$ & & & & & \\
\hline $35-44$ & 9.4 & 9.2 & 11.6 & 8.4 & $<0.0001$ & $\mathrm{PCI}^{\mathrm{a}}$ & 6.3 & 6.8 & 6.9 & 5.9 & 0.1510 \\
\hline $45-54$ & 30.2 & 34.4 & 34.5 & 27.6 & $<0.0001$ & $\mathrm{CABG}^{\mathrm{a}}$ & 1.1 & 1.1 & 0.6 & 1.3 & 0.0030 \\
\hline $55-64$ & 41.0 & 40.7 & 44.1 & 39.6 & 0.0001 & $\begin{array}{l}\text { Coronary } \\
\text { revascb }\end{array}$ & 87.3 & 95.4 & 96.3 & 82.1 & $<0.0001$ \\
\hline $65-74$ & 10.5 & 9.3 & 7.8 & 11.9 & $<0.0001$ & $\frac{\mathrm{PCI}^{\mathrm{b}}}{}$ & 85.9 & 95.1 & 96.2 & 80.0 & $<0.0001$ \\
\hline 75-84 & 5.6 & 4.8 & 1.1 & 7.7 & $<0.0001$ & $\mathrm{CABG}^{\mathrm{b}}$ & 6.5 & 2.5 & 1.4 & 9.3 & $<0.0001$ \\
\hline$>85$ & 2.6 & 0.6 & 0.1 & 4.0 & $<0.0001$ & $\mathrm{CHF}$ & 11.0 & 9.3 & 8.0 & 12.7 & $<0.0001$ \\
\hline \multicolumn{6}{|l|}{ Gender, \% } & $\mathrm{AF}$ & 3.9 & 2.5 & 2.9 & 4.6 & $<0.0001$ \\
\hline Female & 23.8 & 21.5 & 18.1 & 26.7 & $<0.0001$ & Renal disease & 4.3 & 3.1 & 2.5 & 5.4 & $<0.0001$ \\
\hline \multicolumn{6}{|l|}{ Benefit plan, \% } & Stroke/TIA & 6.5 & 4.0 & 2.9 & 8.5 & $<0.0001$ \\
\hline EPO/HMO & 14.8 & 14.9 & 14.3 & 15.0 & 0.7149 & DVT & 0.7 & 0.2 & 0.6 & 0.8 & 0.0682 \\
\hline $\mathrm{PPO}$ & 53.4 & 55.2 & 56.3 & 51.8 & 0.0001 & PAD & 3.5 & 2.8 & 2.4 & 4.2 & $<0.0001$ \\
\hline POS/POS & 8.7 & 9.2 & 9.4 & 8.3 & 0.1856 & \multicolumn{6}{|c|}{ Concomitant medication use, $\%$} \\
\hline with capitation & & & & & & \multirow{2}{*}{\multicolumn{2}{|c|}{\begin{tabular}{|l|l|}
$\begin{array}{l}\text { Beta-adrenergic } \\
\text { antagonists }\end{array}$ & 73.3 \\
\end{tabular}}} & \multirow[t]{2}{*}{76.4} & \multirow[t]{2}{*}{75.2} & \multirow[t]{2}{*}{71.9} & \multirow[t]{2}{*}{$<0.0001$} \\
\hline Comprehensive & 11.1 & 7.8 & 7.0 & 13.4 & $<0.0001$ & & & & & & \\
\hline Other & 8.0 & 9.7 & 8.6 & 7.6 & 0.0200 & \multirow{3}{*}{$\begin{array}{l}\text { Angiotensin } \\
\text { II receptor } \\
\text { antagonists } \\
\end{array}$} & \multirow[t]{3}{*}{7.0} & \multirow[t]{3}{*}{8.0} & \multirow[t]{3}{*}{6.8} & \multirow[t]{2}{*}{6.9} & \multirow[t]{3}{*}{0.3889} \\
\hline Unknown & 4.0 & 3.2 & 4.4 & 4.0 & 0.2326 & & & & & & \\
\hline \multicolumn{6}{|c|}{ Comorbidities, \% } & & & & & & \\
\hline Hypertension & 44.6 & 42.7 & 39.9 & 46.9 & $<0.0001$ & Statins & 77.7 & 80.2 & 81.1 & 75.8 & $<0.0001$ \\
\hline Hyperlipidemia & 53.4 & 52.6 & 55.4 & 52.7 & 0.0276 & $\begin{array}{l}\text { Proton pump } \\
\text { inhibitors }\end{array}$ & 14.8 & 16.2 & 14.9 & 14.6 & 0.3698 \\
\hline Diabetes & 20.6 & 19.0 & 18.3 & 21.9 & 0.0001 & \multirow{2}{*}{$\begin{array}{l}\mathrm{H}_{2} \text {-receptor } \\
\text { antagonists }\end{array}$} & \multirow[t]{2}{*}{4.4} & \multirow[t]{2}{*}{3.3} & \multirow[t]{2}{*}{3.3} & 5.0 & 0.0001 \\
\hline COPD & 7.3 & 5.4 & 4.8 & 8.7 & $<0.0001$ & & & & & & \\
\hline Previous MI & 42.2 & 42.6 & 41.6 & 42.4 & 0.6973 & ACE inhibitors & 49.5 & 52.7 & 52.4 & 47.7 & $<0.0001$ \\
\hline $\begin{array}{l}\text { aIn baseline perio } \\
{ }^{b} \text { During index ho } \\
\mathrm{ACE}=\text { angiotensi } \\
\mathrm{CHF}=\text { chronic he } \\
\text { maintenance orgd }\end{array}$ & $\begin{array}{l}\text { n-convertin } \\
\text { art failure; }\end{array}$ & $y m e ; A C$ & $\begin{array}{l}\text { ute cor } \\
\text { tructive }\end{array}$ & indrom & $=a t r i$ & On; API = & reter & provic & . & $\begin{array}{l}\text { byp } \\
1 O=\end{array}$ & (a) \\
\hline
\end{tabular}

follow-up period (Figure 1), with little variation across treatment groups $(31.1 \%, 30.6 \%$, and $34.8 \%$ among ticagrelor, prasugrel, and clopidogrel users, respectively). Dyspnea was reported in $14.5 \%$ of ticagrelor users, in $13.6 \%$ of prasugrel users, and in $16.5 \%$ of clopidogrel users. Also, heart block was more common in clopidogrel users (15.1\%), followed by ticagrelor (12.2\%) and prasugrel (11.8\%) users. Overall, bradycardia and syncope were more infrequent (4.4\% and 3.9\%, respectively), but they were also more common in clopidogrel users than in ticagrelor and prasugrel users. In addition, major or life-threatening bleeding (7.9\%) and major gastrointestinal bleeding (3.8\%) occurred more commonly in clopidogrel users compared with ticagrelor or prasugrel users. Intracranial bleeding was rare in all groups $(0.3 \%, 0.1 \%$, and $0.2 \%$ among ticagrelor, prasugrel, and clopidogrel users, respectively).
There were significant differences between patients who experienced P2Y12-associated complications and those who did not across all treatment groups (Table 2). On average, those who experienced P2Y12-associated complications were younger, more often male, and had preferred provider organization insurance coverage, and they less often had comprehensive insurance and a history of chronic obstructive pulmonary disease (COPD), coronary artery bypass graft, congestive heart failure, atrial fibrillation, renal disease, and transient ischemic attack. Clopidogrel users who experienced P2Y12-associated complications less often had hypertension and diabetes but more often had hyperlipidemia and deep vein thrombosis during the baseline period. All the ACS patients within each treatment group who experienced P2Y12-associated complications were less often on proton pump inhibitors, while clopidogrel users who experienced P2Y12 complications were more often on 


\section{FIGURE 1 Proportion of ACS Patients Who Experienced P2Y12-Associated Complications Within 12 Months $^{\mathrm{a}}$}

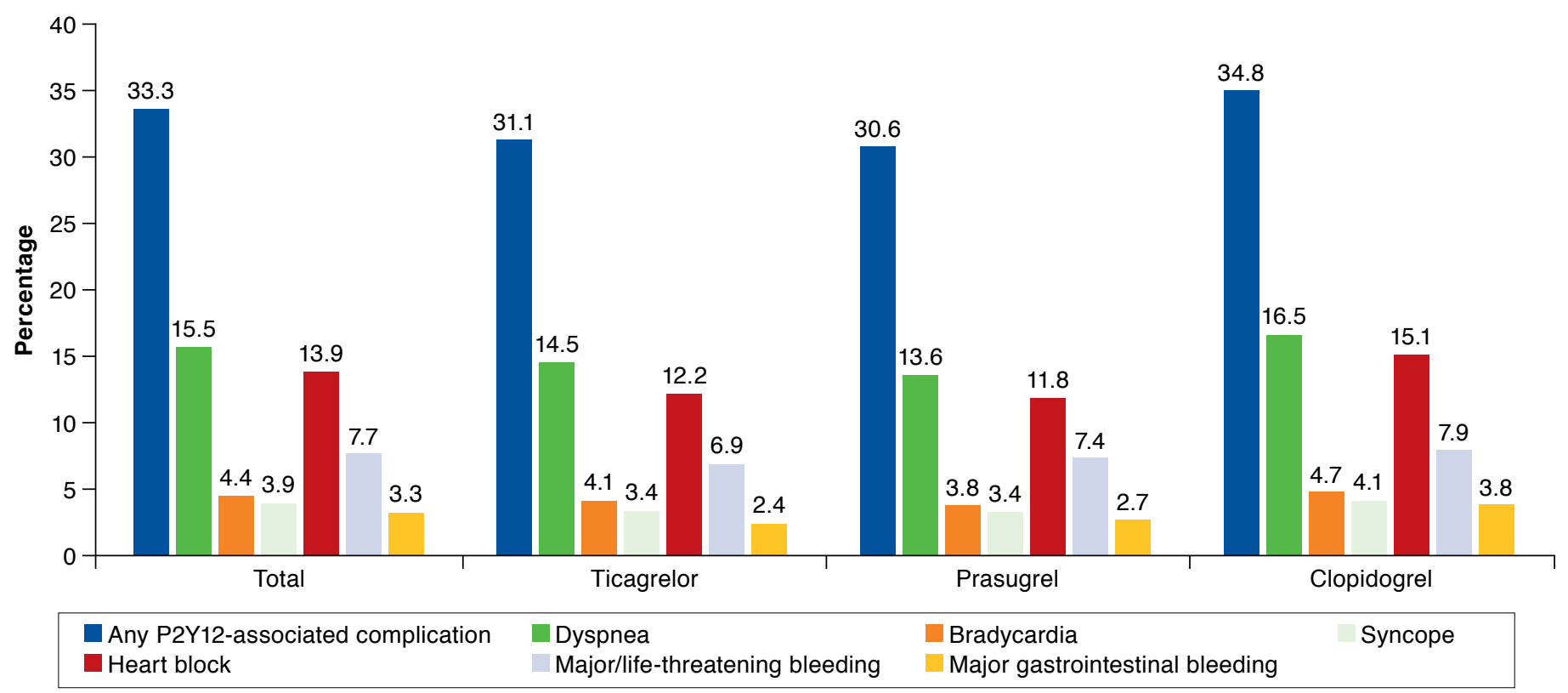

aOther P2Y12-associated complications with rate below $2.0 \%$ include elevated serum creatinine, elevated serum uric acid, and intracranial bleeding/hemorrhage-elevated serum creatinine: total (0.2\%), ticagrelor (0.1\%), prasugrel (0.3\%), and clopidogrel $(0.2 \%)$; elevated serum uric acid: total (2.0\%), ticagrelor (2.0\%), prasugrel (2.2\%), and clopidogrel (1.9\%); intracranial bleeding/hemorrhage: total (0.2\%), ticagrelor (0.3\%), prasugrel (0.1\%), and clopidogrel (0.2\%).

ACS = acute coronary syndrome.

statins and beta-adrenergic antagonists and less often on angiotensin II receptor antagonists and H2-receptor antagonists.

\section{P2Y12 Treatment Changes}

Sixty-eight percent of the study cohort was adherent to P2Y12 APT during the follow-up period (Table 3). Adherence was highest among prasugrel users (73.3\%), followed by ticagrelor (71.4\%) and clopidogrel users (65.6\%). PDC was, on average, $80 \%$ in all patients, with little variation by treatment group (0.82 [SD 0.23], 0.83 [SD 0.23], and 0.78 [SD 0.29] among ticagrelor, prasugrel, and clopidogrel users, respectively). The proportion of ACS patients who were persistent with P2Y12 APT was similar at $78.8 \%$, with slight variation among clopidogrel users (79.5\%), followed by $78.5 \%$ among prasugrel users and $74.5 \%$ among ticagrelor users. A switch in P2Y12 APT was observed more often in ticagrelor (16.9\%) and prasugrel (12.6\%) users than in clopidogrel users (2.1\%).

\section{Health Care Utilization}

Nearly all patients experienced outpatient visits following an ACS event, and a large portion also experienced hospitalizations (20.2\%) and ER visits (30.9\%; Table 3). On average, more patients treated with clopidogrel (22.1\%) had inpatient hospitalizations with longer lengths of stay compared with those patients treated with ticagrelor (17.1\%) or prasugrel (17.1\%). Cardiac events and transfusions were more common in clopidogrel users $(5.7 \%$ and $2.2 \%$, respectively) compared with ticagrelor and prasugrel users.

\section{Associations Between Nonadherence to P2Y12 APT and Health Care Utilization}

On average, patients not adherent to P2Y12 APT experienced all forms of health care utilization more frequently compared with adherent patients, with the exception of outpatient visits and cardiac events (Table 4). After adjusting for demographic and clinical characteristics, we observed that nonadherers had 1.34 (95\% confidence interval $[\mathrm{CI}]=1.25-1.44$ ) times as many hospitalizations, with 1.83 (95\% CI=1.76-1.89) times the length of stay, 1.09 (95\% CI=1.04-1.15) times the number of ER visits, and 1.85 (95\% CI $=1.45-2.37)$ times as many transfusions compared with adherent patients. These trends of association were similar across all treatment groups. Cardiac events differed by adherence group among ticagrelor users, where nonadherent patients had $1.90(95 \% \mathrm{CI}=1.00-3.58)$ times the number of events compared with adherent patients. The mean number of outpatient visits among patients who were not adherent to P2Y12 APT was $8 \%$ lower than that among patients adherent to P2Y12 APT. 


\section{Changes in Treatment Patterns and Incremental Health Care Utilization Due to P2Y12-Associated Complications in Patients with Acute Coronary Syndrome}

TABLE 2 Description of ACS Patients By Experience of P2Y12-Associated Complications

\begin{tabular}{|c|c|c|c|c|c|c|c|c|c|}
\hline & \multicolumn{3}{|c|}{ Ticagrelor Users } & \multicolumn{3}{|c|}{ Prasugrel Users } & \multicolumn{3}{|c|}{ Clopidogrel Users } \\
\hline & $\begin{array}{c}\text { With P2Y12 } \\
\text { Complications }\end{array}$ & $\begin{array}{l}\text { Without P2Y12 } \\
\text { Complications }\end{array}$ & $P$ Value & $\begin{array}{c}\text { With P2Y12 } \\
\text { Complications } \\
\end{array}$ & $\begin{array}{l}\text { Without P2Y12 } \\
\text { Complications } \\
\end{array}$ & $P$ Value & $\begin{array}{c}\text { With P2Y12 } \\
\text { Complications } \\
\end{array}$ & $\begin{array}{l}\text { Without P2Y12 } \\
\text { Complications }\end{array}$ & $P$ Value \\
\hline Age, mean (SD) & $55.7(9.5)$ & $57.7(10.7)$ & $<0.0010$ & $54.4(8.3)$ & $55.5(8.7)$ & $<0.0010$ & $57.2(10.9)$ & $61.4(13.0)$ & $<0.0010$ \\
\hline \multicolumn{10}{|c|}{ Age group, years, $\%$} \\
\hline$<35$ & 0.8 & 1.4 & 0.1620 & 1.1 & 0.6 & 0.1000 & 1.0 & 0.9 & 0.4291 \\
\hline $35-44$ & 10.7 & 8.5 & 0.0857 & 11.8 & 9.0 & 0.0015 & 9.8 & 6.4 & $<0.0010$ \\
\hline $45-54$ & 32.7 & 28.0 & 0.0194 & 35.1 & 33.9 & 0.3970 & 29.8 & 24.2 & $<0.0010$ \\
\hline $55-64$ & 41.5 & 42.6 & 0.6055 & 42.7 & 45.4 & 0.0530 & 41.2 & 37.1 & $<0.0010$ \\
\hline $65-74$ & 10.7 & 10.8 & 0.9399 & 8.5 & 9.0 & 0.5190 & 11.0 & 14.1 & $<0.0010$ \\
\hline $75-84$ & 2.9 & 7.2 & $<0.0010$ & 0.8 & 1.8 & 0.0011 & 4.5 & 10.7 & $<0.0010$ \\
\hline$>85$ & 0.6 & 1.6 & 0.0284 & 0.0 & 0.2 & 0.0166 & 2.7 & 6.7 & $<0.0010$ \\
\hline \multicolumn{10}{|l|}{ Gender, \% } \\
\hline Female & 19.4 & 25.5 & 0.0006 & 16.8 & 22.1 & $<0.0010$ & 24.8 & 32.1 & $<0.0010$ \\
\hline \multicolumn{10}{|c|}{ Medical history, \% } \\
\hline Hypertension & 45.0 & 46.2 & 0.5718 & 42.2 & 43.3 & 0.4188 & 46.3 & 52.9 & $<0.0010$ \\
\hline Hyperlipidemia & 57.1 & 56.1 & 0.6629 & 59.2 & 56.4 & 0.0505 & 56.0 & 53.8 & 0.0137 \\
\hline Diabetes & 19.1 & 20.4 & 0.4420 & 20.1 & 19.7 & 0.6957 & 22.9 & 26.9 & $<0.0010$ \\
\hline COPD & 5.4 & 8.2 & 0.0067 & 4.4 & 7.1 & $<0.0010$ & 7.5 & 13.0 & $<0.0010$ \\
\hline MI & 39.4 & 41.8 & 0.2514 & 41.8 & 43.0 & 0.4243 & 42.2 & 42.5 & 0.7028 \\
\hline Coronary revasc $^{\mathrm{a}}$ & 6.9 & 8.7 & 0.1068 & 7.4 & 8.0 & 0.4474 & 6.8 & 7.6 & 0.0584 \\
\hline $\mathrm{PCI}^{\mathrm{a}}$ & 6.5 & 7.8 & 0.2230 & 7.1 & 7.8 & 0.3612 & 5.8 & 6.2 & 0.3947 \\
\hline $\mathrm{CABG}^{\mathrm{a}}$ & 0.6 & 1.4 & 0.0345 & 0.6 & 0.6 & 0.9910 & 1.3 & 2.1 & 0.0004 \\
\hline Coronary revasc ${ }^{b}$ & 94.7 & 94.7 & 0.9636 & 96.7 & 95.5 & 0.0254 & 82.8 & 80.1 & 0.0001 \\
\hline $\mathrm{PCI}^{\mathrm{b}}$ & 94.5 & 94.3 & 0.7951 & 96.7 & 95.3 & 0.0130 & 80.6 & 77.1 & $<0.0010$ \\
\hline $\mathrm{CABG}^{\mathrm{b}}$ & 2.0 & 2.6 & 0.3422 & 1.5 & 1.8 & 0.5218 & 9.0 & 11.1 & 0.0002 \\
\hline $\mathrm{CHF}$ & 8.1 & 13.9 & $<0.0010$ & 7.4 & 10.9 & $<0.0010$ & 11.0 & 19.0 & $<0.0010$ \\
\hline $\mathrm{AF}$ & 1.5 & 4.9 & $<0.0010$ & 1.9 & 4.2 & $<0.0010$ & 2.8 & 8.5 & $<0.0010$ \\
\hline Renal disease & 2.6 & 5.3 & 0.0007 & 2.1 & 3.8 & 0.0002 & 4.3 & 9.1 & $<0.0010$ \\
\hline TIA & 3.5 & 6.9 & 0.0002 & 3.0 & 3.8 & 0.1057 & 7.5 & 11.1 & $<0.0010$ \\
\hline DVT & 0.3 & 0.4 & 0.6962 & 0.3 & 0.6 & 0.1310 & 0.6 & 1.1 & 0.0044 \\
\hline PAD & 2.3 & 3.6 & 0.0557 & 2.1 & 3.6 & 0.0007 & 3.5 & 6.6 & $<0.0010$ \\
\hline \multicolumn{10}{|c|}{ Medication use, $\%$} \\
\hline $\begin{array}{l}\text { Beta-adrenergic } \\
\text { antagonists }\end{array}$ & 75.5 & 75.5 & 0.9786 & 76.4 & 74.9 & 0.2162 & 73.7 & 70.7 & 0.0001 \\
\hline $\begin{array}{l}\text { Angiotensin II } \\
\text { receptor } \\
\text { antagonists }\end{array}$ & 6.5 & 8.3 & 0.0942 & 7.2 & 7.9 & 0.3765 & 6.5 & 8.7 & $<0.0010$ \\
\hline Statins & 80.6 & 80.2 & 0.8255 & 81.3 & 80.9 & 0.7104 & 77.9 & 73.6 & $<0.0010$ \\
\hline $\begin{array}{l}\text { Proton pump } \\
\text { inhibitors }\end{array}$ & 13.1 & 20.7 & $<0.0010$ & 14.3 & 18.2 & 0.0001 & 13.9 & 18.3 & $<0.0010$ \\
\hline $\begin{array}{l}\text { H2-receptor } \\
\text { antagonists }\end{array}$ & 2.5 & 3.3 & 0.3078 & 2.9 & 3.6 & 0.1466 & 4.5 & 5.8 & 0.0006 \\
\hline ACE inhibitors & 52.4 & 52.1 & 0.9012 & 52.6 & 52.3 & 0.8491 & 48.7 & 47.2 & 0.0967 \\
\hline
\end{tabular}

a In baseline period.

${ }^{b}$ During index hospitalization

$A C E=$ angiotensin-converting enzyme; $A C S=$ acute coronary syndrome; $A F=$ atrial fibrillation; $C A B G=$ coronary artery bypass graft; $C H F=$ chronic heart failure;

$C O P D=$ chronic obstructive pulmonary disease; $D V T=$ deep vein thrombosis; $E P O=$ exclusive provider organization; HMO = health maintenance organization;

$M I=$ myocardial infarction; $P A D=$ peripheral arterial disease; $P C I=$ percutaneous coronary intervention; $P O S=$ point of service, $P P O=$ preferred provider organization; revasc = revascularization; $S D=$ standard deviation; $T I A=$ transient ischemic attack.

\section{Discussion}

This study assessed the proportion of ACS patients who experienced P2Y12-associated complications, adherence and persistence to P2Y12 APT, and its association with health care utilization. We observed adherence to be associated with improved outcomes in a real-world setting using a large U.S. claims database (MarketScan) with 200 million unique patients and a wide national geographic coverage. 


\begin{tabular}{|c|c|c|c|c|}
\hline TABLE 3 & $\begin{array}{l}\text { One-Year T } \\
\text { Health Car } \\
\text { P2Y12-Trea }\end{array}$ & $\begin{array}{l}\text { eatmen } \\
\text { Utilizat } \\
\text { d ACS }\end{array}$ & $\begin{array}{l}\text { atterns } \\
\text { Amor } \\
\text { ients }\end{array}$ & \\
\hline & \multirow[b]{2}{*}{ All Patients } & \multicolumn{3}{|c|}{ Index P2Y12 Treatment } \\
\hline & & $\begin{array}{l}\text { Ticagrelor } \\
\text { Users }\end{array}$ & $\begin{array}{l}\text { Prasugrel } \\
\text { Users }\end{array}$ & $\begin{array}{c}\text { Clopidogrel } \\
\text { Users }\end{array}$ \\
\hline $\begin{array}{l}\text { Adherence }(\mathrm{PDC}), \\
\text { mean (SD) }\end{array}$ & $\begin{array}{c}0.80 \\
(0.267) \\
\end{array}$ & $\begin{array}{c}0.82 \\
(0.232) \\
\end{array}$ & $\begin{array}{c}0.83 \\
(0.230) \\
\end{array}$ & $\begin{array}{c}0.78 \\
(0.285) \\
\end{array}$ \\
\hline Adherence, ${ }^{\mathrm{b}} \%$ & 68.3 & 71.4 & 73.3 & 65.6 \\
\hline Persistence, ${ }^{c} \%$ & 78.8 & 74.5 & 78.5 & 79.5 \\
\hline Switching, ${ }^{\mathrm{d}} \%$ & 6.4 & 16.9 & 12.6 & 2.1 \\
\hline Hospitalizations, $\%$ & 20.2 & 17.1 & 17.1 & 22.1 \\
\hline Mean (SD) & $\begin{array}{c}1.41 \\
(0.940)\end{array}$ & $\begin{array}{c}1.33 \\
(0.964)\end{array}$ & $\begin{array}{c}1.36 \\
(0.849) \\
\end{array}$ & $\begin{array}{c}1.44 \\
(0.967) \\
\end{array}$ \\
\hline Median (IQR) & $\begin{array}{c}1 \\
(1-2)\end{array}$ & $\begin{array}{c}1 \\
(1-1)\end{array}$ & $\begin{array}{c}1 \\
(1-1)\end{array}$ & $\begin{array}{c}1 \\
(1-2)\end{array}$ \\
\hline \multicolumn{5}{|c|}{ Hospital length of staye } \\
\hline Mean (SD) & $\begin{array}{c}5.6 \\
(8.6) \\
\end{array}$ & $\begin{array}{c}5.2 \\
(10.7) \\
\end{array}$ & $\begin{array}{c}5.0 \\
(7.6)\end{array}$ & $\begin{array}{c}5.9 \\
(8.6) \\
\end{array}$ \\
\hline Median (IQR) & $\begin{array}{c}3 \\
(2-6)\end{array}$ & $\begin{array}{c}3 \\
(2-5)\end{array}$ & $\begin{array}{c}3 \\
(1-5)\end{array}$ & $\begin{array}{c}3 \\
(2-7)\end{array}$ \\
\hline $\begin{array}{l}\text { Emergency room } \\
\text { visits, \% }\end{array}$ & 30.9 & 28.9 & 29.8 & 31.6 \\
\hline Mean (SD) & $\begin{array}{c}1.83 \\
(1.778) \\
\end{array}$ & $\begin{array}{c}1.78 \\
(1.948)\end{array}$ & $\begin{array}{c}1.76 \\
(1.258) \\
\end{array}$ & $\begin{array}{c}1.87 \\
(1.937)\end{array}$ \\
\hline Median (IQR) & $\begin{array}{c}1 \\
(1-2)\end{array}$ & $\begin{array}{c}1 \\
(1-2)\end{array}$ & $\begin{array}{c}1 \\
(1-2)\end{array}$ & $\begin{array}{c}1 \\
(1-2)\end{array}$ \\
\hline Outpatient visits, \% & 99.6 & 99.9 & 99.5 & 99.5 \\
\hline Mean (SD) & $\begin{array}{c}24.19 \\
(21.185) \\
\end{array}$ & $\begin{array}{c}23.80 \\
(20.126) \\
\end{array}$ & $\begin{array}{c}23.34 \\
(18.825) \\
\end{array}$ & $\begin{array}{c}24.63 \\
(22.303) \\
\end{array}$ \\
\hline Median (IQR) & $\begin{array}{c}18 \\
(10-32)\end{array}$ & $\begin{array}{c}17 \\
(10-33) \\
\end{array}$ & $\begin{array}{c}17 \\
(10-31)\end{array}$ & $\begin{array}{c}18 \\
(10-32)\end{array}$ \\
\hline Cardiac events, $\%$ & 5.1 & 3.9 & 4.2 & 5.7 \\
\hline Mean (SD) & $\begin{array}{c}1.07 \\
(0.303)\end{array}$ & $\begin{array}{c}1.05 \\
(0.312)\end{array}$ & $\begin{array}{c}1.08 \\
(0.361) \\
\end{array}$ & $\begin{array}{c}1.07 \\
(0.281)\end{array}$ \\
\hline Median (IQR) & $\begin{array}{c}1 \\
(1-1)\end{array}$ & $\begin{array}{c}1 \\
(1-1)\end{array}$ & $\begin{array}{c}1 \\
(1-1)\end{array}$ & $\begin{array}{c}1 \\
(1-1)\end{array}$ \\
\hline Transfusions, \% & 2.0 & 1.7 & 1.8 & 2.2 \\
\hline Mean (SD) & $\begin{array}{c}1.15 \\
(0.475) \\
\end{array}$ & $\begin{array}{c}1.28 \\
(0.752) \\
\end{array}$ & $\begin{array}{c}1.17 \\
(0.378) \\
\end{array}$ & $\begin{array}{c}1.13 \\
(0.468)\end{array}$ \\
\hline Median (IQR) & $\begin{array}{c}1 \\
(1-1)\end{array}$ & $\begin{array}{c}1 \\
(1-1) \\
\end{array}$ & $\begin{array}{c}1 \\
(1-1) \\
\end{array}$ & $\begin{array}{c}1 \\
(1-1)\end{array}$ \\
\hline
\end{tabular}

aPDC was calculated as the ratio of number of days P2Y12 APT was supplied and number of days in the follow-up period.

${ }^{b}$ Patients with $P D C \geq 80 \%$ were considered adherent to P2Y12 APT.

cContinuation or discontinuation of P2Y12 APT where the gap in therapy of at least 30 days was considered discontinuation and nonpersistent to therapy.

dPatients on current P2Y12 APT that they discontinued for 30 days or more and replaced by another antiplatelet drug.

${ }^{e}$ Length of stay (in days) was defined as the duration between hospital admission and discharge, among only those with at least 1 hospitalization.

$A C S=$ acute coronary syndrome; $A P T=$ antiplatelet therapy; $I Q R=$ interquartile range; $P D C=$ proportion of days covered; $S D=$ standard deviation.

As expected, a smaller proportion of ACS patients had initiated ticagrelor within 30 days of their index event than those with an index APT of prasugrel and clopidogrel, a finding consistent with Kim et. al. (2017); however, this study focused on ACS patients treated with percutaneous coronary intervention (PCI). ${ }^{21}$ This finding may be a result of low U.S. market penetration of ticagrelor close to the timing of drug launch in August 2011 (not long before this analysis initiated inclusion of ACS patients, from January 1, 2012, onwards). Our study findings indicate that a large proportion of ACS patients experienced P2Y12-associated complications and that adherence to P2Y12 APT among ACS patients was suboptimal and likely contributed to increased health care utilization.

In our study, we observed that a greater proportion of patients with index clopidogrel APT experienced dyspnea and several other P2Y12-associated complications compared with those patients using ticagrelor. These observations differ from the findings in the PLATO trial. ${ }^{12}$ This difference may be because patients and physicians were aware of common complications and potential side effects associated with ticagrelor (by the time it was in use) so were less likely to use health care services when they experienced transient or mild-to-moderate side effects (e.g., dyspnea). While patients may have still experienced these side effects, they would not have been captured in the commercial claims database if they went untreated.

Patients who did not experience any P2Y12-associated complications more often had a higher proportion of certain baseline comorbidities, such as COPD, congestive heart failure, and atrial fibrillation, compared with those patients who did experienced P2Y12 complications. This situation may also have led to underreporting of side effects if, for instance, ACS patients with COPD (for whom dyspnea is the most commonly occurring event) experienced dyspnea related to P2Y12 APT and did not report it.

Consistent with a previous report, ${ }^{12}$ the rates of intracranial bleeding and hemorrhage in our study were 0.3\% among ticagrelor users compared with $0.2 \%$ among clopidogrel users. However, we observed that $7.4 \%$ of ACS patients with index prasugrel treatment experienced major or life-threatening bleeding, with most of the patients switching to another APT, which was inconsistent with the TRITON-TIMI trial. ${ }^{8}$ A similar proportion $(7.9 \%)$ of patients with index clopidogrel treatment experienced the same complication but did not switch to another APT. Switching to another APT was common among ticagrelor users (16.9\%) compared with clopidogrel users $(2.1 \%)$, even though a higher proportion of clopidogrel users experienced P2Y12-associated complications.. This high switching rate among ticagrelor users was consistent with the ticagrelor withdrawal rate and ticagrelor switching rate from other published studies, ${ }^{16,22}$ although 1 of the studies evaluated the withdrawal rates over only a 1-month follow-up period in contrast to the 1-year follow-up assessed in our study. ${ }^{16}$ 
Changes in Treatment Patterns and Incremental Health Care Utilization Due to

P2Y12-Associated Complications in Patients with Acute Coronary Syndrome

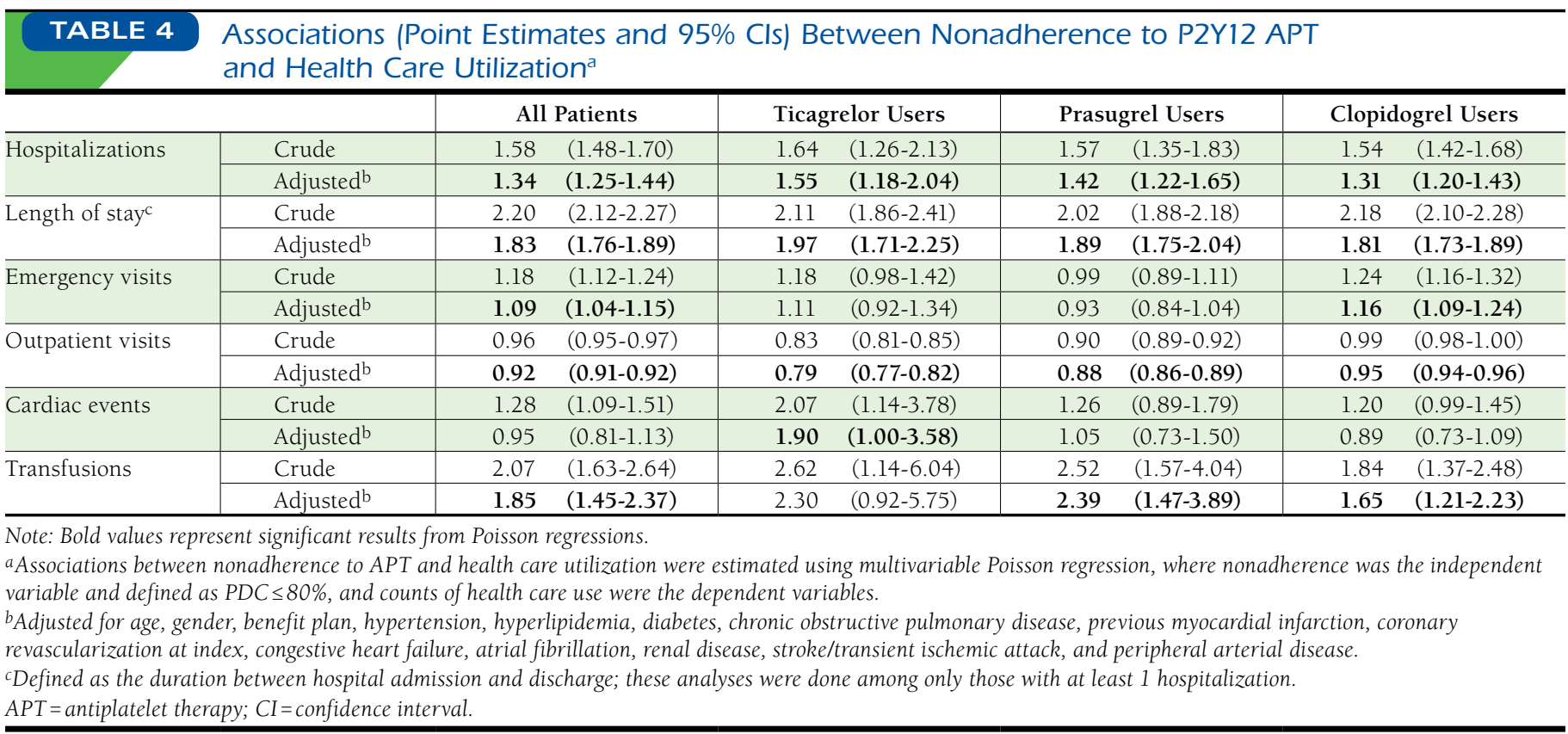

Following the trend of the frequency of reported side effects, which was highest among clopidogrel users and lowest among prasugrel users, the converse was true when considering adherence. The proportion of patients adherent to prasugrel during the follow-up period was highest and was lowest among clopidogrel users. This study finding is consistent with a previous report of higher adherence rates in prasugrel users compared with ticagrelor users. ${ }^{22}$ The proportion of ACS patients adherent to clopidogrel was highly consistent with a 2011 study by Zhu et al. ${ }^{23}$ Nordstrom et al. (2013) reported an adherence rate of $78.8 \%$ among ACS patients with $\mathrm{PCI},{ }^{24}$ which is higher than that found in our study (68\%). This discrepancy may be because the Nordstrom et al. study used medication possession ratio (MPR) to report adherence, while we used PDC estimates. MPR has been associated with overestimation of the true rate of medication adherence, while PDC has provided more conservative estimates of adherence. ${ }^{19}$ However, Nordstrom et al. reported lower adherence in both treatment groups than what we observed in our study, ${ }^{24}$ most likely because of differences in databases (provider-based vs. insurance-based) and/or study cohorts (ACS with PCI vs. ACS).

Patients with index ticagrelor or prasugrel treatment in our study had similar rates of inpatient hospitalizations to that reported in the 2015 study conducted by Simeone et al. ${ }^{22}$ Nonadherence to all P2Y12 APT was significantly associated with higher rates of inpatient hospitalizations and increased length of hospital stay. Likewise, ACS patients not adherent to ticagrelor were more likely to experience cardiac events compared with adherent patients, and patients not adherent to prasugrel or clopidogrel were also more likely to undergo blood transfusions compared with adherent patients.

These adherence findings highlight the need to improve adherence to P2Y12 APT, since poor adherence is associated with increased health care utilization and increased cardiac events among ticagrelor users. ${ }^{25,26}$ These findings should be interpreted with caution given the uncertainty in the direction and nature of the association between nonadherence and increased health care utilization. We hypothesized that the ACS patients on P2Y12 APT may be discontinuing, instead of adhering to, their regimens because of the associated complications that, in turn, lead to increased health care utilization. However, it is possible that P2Y12-associated complications may more likely be captured and reported in ACS patients who have frequent hospitalizations or that sicker patients are less likely to adhere to therapies.

Disease management strategies that may improve adherence and reduce treatment-associated adverse events through individualized patient care should be developed and practiced among high-risk patients. Alternative secondary treatment options that can further reduce cardiovascular risk with fewer side effects (and presumably better adherence) should be considered. ${ }^{27-30}$

\section{Limitations}

There are some limitations to this study that need to be considered. While the MarketScan database used in this study 
represents a large number of commercially insured individuals in the United States with the advantages of integrated health care data, it is not without disadvantages. Although there is broad U.S. geographic coverage in these data, the southern region is overrepresented and may limit the generalizability of findings to the entire U.S. population. Such biases might encompass differences in disease prevalence and practice patterns by regions. The study population is a select sample of patients observed in a commercial claims database and likely is younger than the wider target population, so there is a possibility of underestimated event rates.

Also, there are certain limitations because of the observational nature of the study, including risk of bias by indication, and unmeasured residual confounding, including race and ethnicity information. In addition, we did not have information on medication consumption, but we assumed that calculations of medication adherence and persistence provided reasonable estimates of true treatment patterns.

Finally, date of death could not be captured, and the sample was restricted to patients with 12 months of follow-up, so results may not be applicable to patients who died during the 12 months after ACS discharge.

\section{Conclusions}

In this observational study, one third of the ACS patients treated with P2Y12 APT experienced at least 1 complication associated with P2Y12, the most common of which were dyspnea, heart block, and major or life-threatening bleeding. Adherence to P2Y12 APT was suboptimal during the 1-year follow-up period, and better adherence was highly associated with lower inpatient hospitalizations, shorter length of hospital stay, and fewer transfusions. Increased adherence to secondary prevention therapy among high-risk patients is critical. Disease management strategies to improve adherence and reduce treatment-associated adverse events through individualized patient care, alternative secondary treatment options, and physician awareness should be designed, implemented, and sustained.

\section{Authors}

AMI VYAS, PhD, MS, Department of Epidemiology, School of Public Health, Rutgers University, Piscataway, New Jersey; LORI D. BASH, PhD, MPH, and MEHUL D. PATEL, PhD, Merck E Co., Kenilworth, New Jersey; and ROSS J. SIMPSON, JR., MD, PhD, Department of Medicine, University of North Carolina, Chapel Hill.

AUTHOR CORRESPONDENCE: Ami Vyas, PhD, MS, University of Rhode Island College of Pharmacy, Dept. of Pharmacy Practice, 7 Greenhouse Rd., Kingston, RI 02881. Tel.: 401.874.7255;

E-mail: avyas@uri.edu.

\section{DISCLOSURES}

Data analysis was conducted by Merck \& Co., the manufacturer of vorapaxar (ZONTIVITY). At the time of this study, Vyas was an employee of Rutgers University, which received grant funding from Merck \& Co. for this study, and is now employed with the University of Rhode Island. Patel was employed by Symphony Solutions and the University of North Carolina during the drafting and revising of the manuscript. Bash is employed by Merck \& Co. Simpson received consulting fees from Merck \& Co. for work on this study and has received fees for research from Amgen and Pfizer.

Study concept and design were contributed by Vyas, Bash, Patel, and Simpson. Patel took the lead in data collection, assisted by the other authors. All the authors contributed equally to data analysis and manuscript preparation.

The abstract for this study was presented as a poster at the American Heart Association Scientific Sessions 2016; November 12-16, 2016; New Orleans, Louisiana.

\section{REFERENCES}

1. World Health Organization. Cardiovascular diseases (CVDs). Fact sheet Updated May 2017. Available at: http://www.who.int/mediacentre/factsheets/ fs317/en/. Accessed July 7, 2017.

2. Go AS, Mozaffarian D, Roger VL, et al. Heart disease and stroke statistics-2014 update: a report from the American Heart Association. Circulation. 2014;129(3):e28-e292.

3. Amsterdam EA, Wenger NK, Brindis RG, et al. 2014 AHA/ACC guideline for the management of patients with non-ST-elevation acute coronary syndrome: a report of the American College of Cardiology/ American Heart Association Task Force on Practice Guidelines. Circulation. 2014;130(25):2354-94.

4. O'Gara PT, Kushner FG, Ascheim DD, et al. 2013 ACCF/AHA guideline for the management of ST-elevation myocardial infarction: a report of the American College of Cardiology Foundation/American Heart Association Task Force on Practice Guidelines. Circulation. 2013;127(4):e362-425.

5. Angiolillo DJ, Guzman LA, Bass TA. Current antiplatelet therapies: benefits and limitations. Am Heart J. 2008;156(2):S3-S9.

6. Yusuf S, Zhao F, Mehta SR, et al. Effects of clopidogrel in addition to aspirin in patients with acute coronary syndromes without ST-segment elevation. N Engl J Med. 2001;345(7):494-502.

7. Bhatt DL, Fox KA, Hacke W, et al. Clopidogrel and aspirin versus aspirin alone for the prevention of atherothrombotic events. $N$ Engl J Med. 2006;354(16):1706-17.

8. Wiviott SD, Braunwald E, McCabe $\mathrm{CH}$, et al. Prasugrel versus clopidogrel in patients with acute coronary syndromes. $N$ Engl J Med. 2007;357(20):2001-15.

9. U.S. Food and Drug Administration. Effient (prasugrel) acute coronary syndromes managed by percutaneous coronary intervention. Cardiovascular and Renal Drugs Advisory Committee briefing document. February 3, 2009. Available at: http://www.fda.gov/ downloads/AdvisoryCommittees/CommitteesMeetingMaterials/Drugs/ CardiovascularandRenalDrugsAdvisoryCommittee/ucm 129219.pdf. Accessed July 8, 2017.

10. Serebruany VL. The TRITON versus PLATO trials: differences beyond platelet inhibition. Thromb Haemost. 2010;103(2):259-61.

11. Spinler SA, Rees C. Review of prasugrel for the secondary prevention of atherothrombosis. J Manag Care Pharm. 2009;15(5):383-95. Available at: http://www.jmcp.org/doi/10.18553/jmcp.2009.15.5.383.

12. Wallentin L, Becker RC, Budaj A, et al. Ticagrelor versus clopidogrel in patients with acute coronary syndromes. N Engl J Med. 2009;361(11):1045-57.

13. Bonaca MP, Bhatt DL, Cohen M, et al. Long-term use of ticagrelor in patients with prior myocardial infarction. N Engl J Med. 2015;372(19):1791-800. 


\section{Changes in Treatment Patterns and Incremental Health Care Utilization Due to P2Y12-Associated Complications in Patients with Acute Coronary Syndrome}

14. BRILINTA (ticagrelor) tablets, for oral use. AstraZeneca. September 2015. Available at: http://www.accessdata.fda.gov/drugsatfda_docs/ label/2015/022433s017lbl.pdf. Accessed July 8, 2017.

15. European Medicines Agency, Committee for Medicinal Products for Human Use. Assessment report: Brilique. Procedure No. EMEA/H/ C/001241/0029/G. December 17, 2015. Available at: http://www.ema.europa eu/docs/en_GB/document_library/EPAR___Assessment_Report_-_Variation/ human/001241/WC500203874.pdf. Accessed July 8, 2017.

16. Gaubert M, Laine M, Richard T, et al. Effect of ticagrelor-related dyspnea on compliance with therapy in acute coronary syndrome patients. Int $J$ Cardiol. 2014;173(1):120-21.

17. Hansen LG, Chang S. Health research data for the real world: the MarketScan databases. White paper. July 2011. Truven Health Analytics. Available at: http://truvenhealth.com/portals/0/assets/PH_11238_0612_ TEMP_MarketScan_WP_FINAL.pdf. Accessed July 8, 2017.

18. Kalyanasundaram A, Lincoff AM; Medscape. Managing adverse effects and drug-drug interactions of antiplatelet agents. Nat Rev Cardiol. 2011;8(10):592-600

19. Nau DP. Proportion of days covered (PDC) as a preferred method of measuring medication adherence. Pharmacy Quality Alliance. Available at: http://www.pqaalliance.org/images/uploads/files/PQA\%20PDC\%20vs\%20 \%20MPR.pdf. Accessed July 8, 2017.

20. Burke JP, Sander S, Shah H, Zarotsky V, Henk H. Impact of persistence with antiplatelet therapy on recurrent ischemic stroke and predictors of nonpersistence among ischemic stroke survivors. Curr Med Res Opin. 2010;26(5):1023-30.

21. Kim K, Lee TA, Touchette DR, et al. Contemporary trends in oral antiplatelet agent use in patients treated with percutaneous coronary intervention for acute coronary syndrome. J Manag Care Spec Pharm. 2017;23(1):57-63. Available at: http://www.jmcp.org/doi/10.18553/jmcp.2017.23.1.57.
22. Simeone JC, Molife C, Marrett E, et al. One-year post-discharge resource utilization and treatment patterns of patients with acute coronary syndrome managed with percutaneous coronary intervention and treated with ticagrelor or prasugrel. Am J Cardiovasc Drugs. 2015;15(5):337-50.

23. Zhu B, Zhao Z, McCollam P, et al. Factors associated with clopidogrel use, adherence, and persistence in patients with acute coronary syndromes undergoing percutaneous coronary intervention. Curr Med Res Opin. 2011;27(3):633-41.

24. Nordstrom BL, Simeone JC, Zhao Z, et al. Adherence and persistence with prasugrel following acute coronary syndrome with percutaneous coronary intervention. Am J Cardiovasc Drugs. 2013;13(4):263-71.

25. Mehran R, Baber U, Steg PG, et al. Cessation of dual antiplatelet treatment and cardiac events after percutaneous coronary intervention (PARIS): 2-year results from a prospective observational study. Lancet. 2013;382(9906):1714-22

26. Desai NR, Choudhry NK. Impediments to adherence to post myocardial infarction medications. Curr Cardiol Rep. 2013;15(1):322.

27. Bonaca MP, Scirica BM, Braunwald E, et al. New ischemic stroke and outcomes with vorapaxar versus placebo: results from the TRA $2{ }^{\circ} \mathrm{P}$-TIMI 50 trial. J Am Coll Cardiol. 2014;64(22):2318-26.

28. Magnani G, Bonaca MP, Braunwald E, et al. Efficacy and safety of vorapaxar as approved for clinical use in the United States. J Am Heart Assoc. 2015;4(3): 001505 .

29. Bohula EA, Aylward PE, Bonaca MP, et al. Efficacy and safety of vorapaxar with or without a thienopyridine for secondary prevention in patients with previous myocardial infarction and no history of stroke or transient ischemic attack: results from TRA $2{ }^{\circ} \mathrm{P}-\mathrm{TIMI} 50$. Circulation. 2015;132(20):1871-79.

30. Morrow DA, Braunwald E, Bonaca MP, et al. Vorapaxar in the secondary prevention of atherothrombotic events. N Engl J Med. 2012;366(15):1404-13. 
Changes in Treatment Patterns and Incremental Health Care Utilization Due to P2Y12-Associated Complications in Patients with Acute Coronary Syndrome

\begin{tabular}{|c|c|}
\hline \multicolumn{2}{|c|}{$\begin{array}{l}\text { APPENDIX A ICD-9-CM Codes for P2Y12- } \\
\text { Associated Complications }\end{array}$} \\
\hline P2Y12 Complications & ICD-9-CM Codes \\
\hline Dyspnea & $786.0 x$ \\
\hline Bradycardia & 427.89 \\
\hline Syncope & 780.2 \\
\hline Heart block & 426.xx, 427.xx \\
\hline Elevated serum creatinine & $794.4 x$ \\
\hline Elevated serum uric acid & $790.6 x$ \\
\hline Intracranial bleeding/hemorrhage & $\begin{array}{l}\text { 430.xx, 431.xx, 432.0x, 432.1x, } \\
432.9 x\end{array}$ \\
\hline Major/life-threatening bleeding & $\begin{array}{l}\text { 430.xx-432.xx, 578.xx, 719.1x, } \\
423.0 x, 599.7 x, 626.2 x, 626.6 x, \\
626.8 x, 627.0 x, 627.1 x, 786.3 x \\
784.7 x, 459.0 x\end{array}$ \\
\hline Major gastrointestinal bleeding & 531.xx-535.xx, 578.9x \\
\hline
\end{tabular}




\section{APPENDIX B Patient Selection Flowchart}

Patients who were discharged from an ACS hospitalization (index date) $N=143,238$

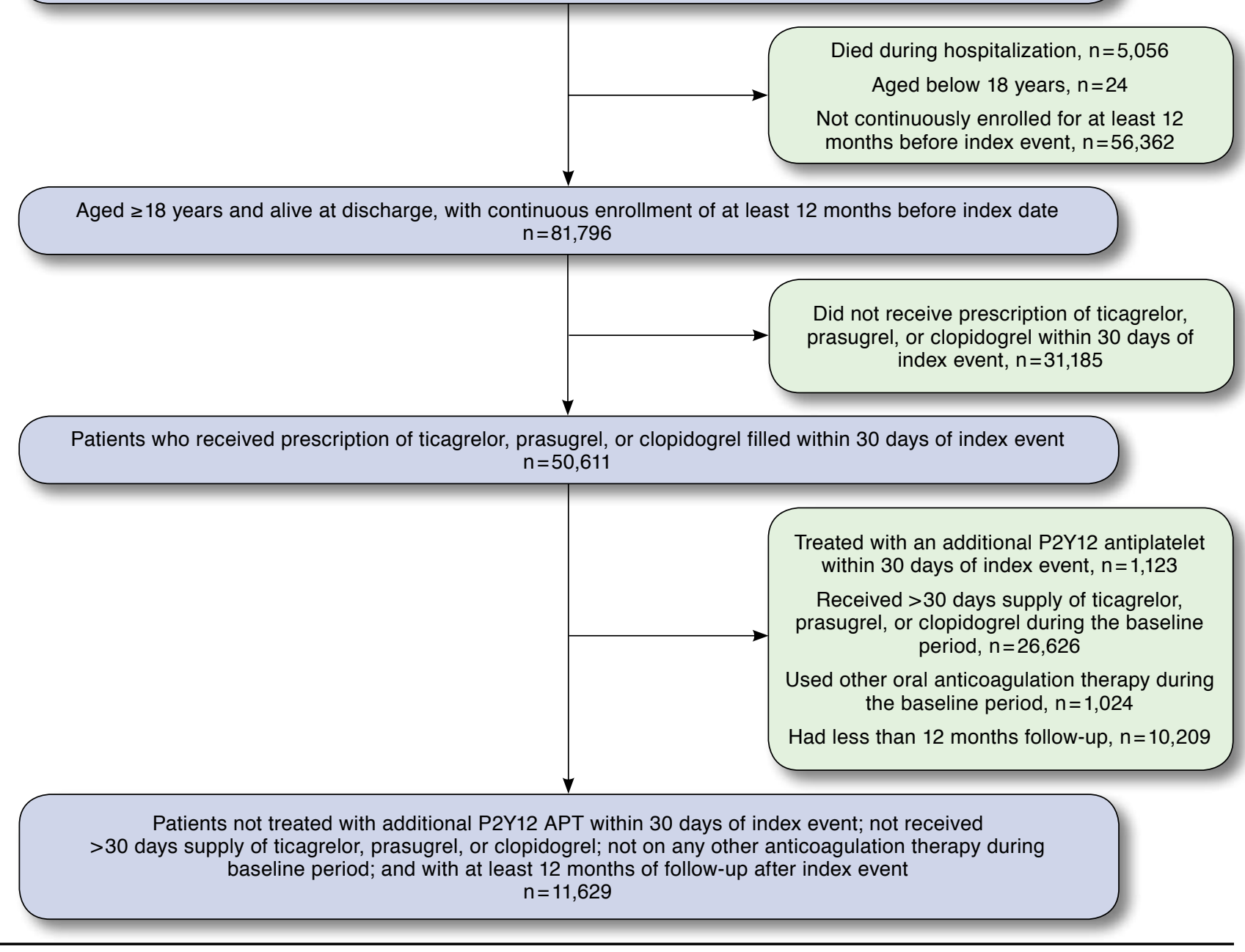

